\title{
Hypoxic regulation of EDN1, EDNRA, EDNRB, and ECE1 gene expressions in ERN1 knockdown U87 glioma cells
}

Dmytro O. Minchenko, ${ }^{1,2}$, Daria O. Tsymbal ${ }^{1}$, Olena O. Riabovol ${ }^{1}$, Yuliia M. Viletska ${ }^{1}$, Yuliia O. Lah anovska ${ }^{1}$, Myroslava Y. Sliusar ${ }^{1}$, Borys H. Bezrodn Y I ${ }^{2}$, Oleksandr H. Minchenko

\author{
${ }^{1}$ Palladin Institute of Biochemistry, National Academy of Sciences of Ukraine, Kyiv, Ukraine; \\ ${ }^{2}$ National Bohomolets Medical University, Kyiv, Ukraine \\ E-mail:ominchenko@yahoo.com
}

Objective. The aim of the present investigation was to study the effect of hypoxia on the expression of genes encoding endothelin-1 (EDN1) and its cognate receptors (EDNRA and EDNRB) as well as endothelin converting enzyme 1 (ECE1) in U87 glioma cells in response to inhibition of endoplasmic reticulum stress signaling mediated by ERN1/IRE1 (endoplasmic reticulum to nucleus signaling 1) for evaluation of their possible significance in the control of glioma growth through ERN1 and hypoxia.

Methods. The expression level of EDN1, EDNRA, EDNRB, and ECE1 genes as well as microRNA miR-19, miR-96, and miR-206 was studied in control and ERN1 knockdown U87 glioma cells under hypoxia by quantitative polymerase chain reaction.

Results. It was shown that the expression level of EDN1, EDNRA, EDNRB, and ECE1 genes was up-regulated in ERN1 knockdown glioma cells in comparison with the control glioma cells, being more significant for endothelin-1. We also observed down-regulation of microRNA miR-206, miR-96, and miR-19a, which have specific binding sites in mRNA EDN1, EDNRA, and EDNRB, correspondingly, and can participate in posttranscriptional regulation of these mRNA expressions. Furthermore, inhibition of ERN1 endoribonuclease lead to up-regulation of EDNRA and ECE1 gene expressions and down-regulation of the expression level of EDN1 and EDNRB genes in glioma cells. Thus, the expression of EDNRA and ECE1 genes is regulated by ERN1 endoribonuclease, but $E D N 1$ and $E D N R B$ genes preferentially by ERN1 protein kinase. We have also shown that hypoxia enhanced the expression of EDN1, EDNRA, and ECE1 genes and that knockdown of ERN1 signaling enzyme function significantly modified the response of all studied gene expressions to hypoxia. Thus, effect of hypoxia on the expression level of EDN1 and ECE1 genes was significantly or completely reduced in ERN1 knockdown glioma cells since the expression of EDNRA gene was down-regulated under hypoxia. Moreover, hypoxia is induced the expression of EDNRB gene in ERN1 knockdown glioma cells.

Conclusions. Results of this investigation demonstrate that ERN1 knockdown significantly increased the expression of endothelin-1 and its receptors as well as ECE1 genes by different mechanisms and that all studied gene expressions were sensitive to hypoxia. It is possible that hypoxic regulation of the expression of these genes is a result of complex interaction of variable ERN1 related transcription and regulatory factors with HIF1A and possibly contributed to the control of glioma growth.

Key words: hypoxia, mRNA expression, EDN1, EDNRA, EDNRB, ECE1, microRNA, ERN1 knockdown, U87 glioma cells

Corresponding author: Oleksandr H. Minchenko, Prof. Department of Molecular Biology, Palladin Institute of Biochemistry, National Academy of Sciences of Ukraine, Leontovycha 9, Kyiv 01030, Ukraine; e-mail: ominchenko@yahoo.com. 
The peptide hormone endothelin-1 (ET-1; EDN1) and its cognate receptors (EDNRA and EDNRB) play an important role in the maintenance of vascular tone, but they are also localized to non-vascular structures including epithelial cells, glia and neurons and play multiple, complex roles (Dashwood and Loesch 2009; Khimji and Rockey 2010; Stow et al. 2011). They also have co-mitogenic activity, potentiating the effects of other growth factors such as PDGF and have been linked to variable diseases including diabetes, traumatic shock and tumorigenesis (Minchenko et al. 1999, 2003; Dashwood and Loesch 2009; Khimji and Rockey 2010; Stow et al. 2011; Palmer et al. 2012; Cook et al. 2015; Dojo Soeandy et al. 2019). There is also a data that EDN1 is a neuropeptide, which is implicated in a number of neural circuits where its transmitter affects range from a role in pain and temperature control to its action on the hypothalamo-neurosecretory system (Dashwood and Loesch 2009). Recently, it has been shown that levels of mRNAs encoding EDN1 and its receptors, known to be elevated in amyotrophic lateral sclerosis, were sharply increased by knockdown of C9 (C9ORF72) gene, which encodes a protein that functions in control of endothelin and glutamate signaling (Fomin et al. 2018). There are also data indicating that melanopsin and clock genes are regulated by light and endothelin in the zebrafish ZEM-2S cell line through interleukin 3 regulated nuclear factor (NFIL3/E4BP4) binding to the promoters and consequent inhibition of gene expressions (Farhat et al. 2009). Differential and tissue-specific production of EDN1 must be tightly regulated in order to preserve these biologically diverse actions.

It is interesting to note that palmitic acid increases ET-1 expression in endothelial cells through the induction of endoplasmic reticulum stress and the activation of protein kinase $\mathrm{C}$, providing novel mechanistic insights into the pathogenesis of obesity-associated hypertension and cardiovascular diseases including atherosclerosis (Zhang et al. 2018). The primary mechanism thought to control EDN1 bioavailability is the rate of transcription from the EDN1 gene. Studies conducted on a variety of cell types have identified key transcription factors including HIF that govern EDN1 expression (Aversa et al. 1997; Minchenko and Caro 2000; Stow et al. 2011). In addition, other mechanisms involved in epigenetic regulation and mRNA stability have emerged as important processes for regulated EDN1 expression.

The endothelin receptor type A is associated with guanine-nucleotide-binding $(\mathrm{G})$ proteins and this coupling activates a phosphatidylinositol-calcium second messenger system. This receptor plays a role in potent and long-lasting vasoconstriction and cell proliferation. Thus, Arabanian et al. (2018) have shown that overexpression of EDNRA led to increased proliferation and resistance to apoptosis of bone marrow cells in vitro and that Meisl binds to the EDNRA promoter region, suggesting a regulatory role for Meis1 in endothelin-1 receptor type A expression and in Hoxa9/Meis1-driven leukemogenesis. Furthermore, the endothelin axis (EDN1 and EDN1 receptor A) is involved in cellular growth, differentiation, invasiveness, and tumor progression in several cancers and polymorphisms of these genes is increased the risk of developing papillary thyroid cancer (Aydin et al. 2019). Recently, it has been observed an abnormal expression of endothelin receptor type B (EDNRB) in hepatocellular carcinoma and confirmed its potential clinical significance (Zhang et al. 2019). Thus, endothelin-1 receptor blockade is new possible therapeutic approach in multiple myeloma, because the majority of multiple myeloma cell lines and primary malignant plasma cells express high levels of EDNRA and EDNRB and release EDN1 (Russignan et al. 2017).

The endothelin-converting enzyme-1 (ECE1) is involved in the proteolytic processing of endothelin precursor to biologically active peptide. Active EDN1 has a short half-life, so its biological effects are completely dependent on its enzymatic activation by ECE1. There are data that expression of ECE1 gene is elevated in several tumors and cancer cell lines and that this protein has relation to cancer cell proliferation and invasiveness (Niechi et al. 2015; Tapia and Niechi 2019). At the same time, ECE1 participates in the development of Furthermore, Miners and Love (2017) have shown that endothelin-converting enzymes degrade alpha-synuclein and are reduced in dementia with Lewy bodies. Thus, it participates in the metabolism of $a$-synuclein that could contribute to the development and progression of dementia with Lewy bodies. It is interesting to note that miR199a-5p, which negatively regulated ECE1, and silencing the ECE1 gene are protected the rat spinal cord injury after ischemia-reperfusion (Bao et al. 2018). Therefore, we demonstrated that miR-199a-5p might protect the spinal cord against ischemia-reperfusion-induced injury by negatively regulating the ECE1. The ECE1 gene is hypoxia responsible and, in its promoter and intron regions, there were identified HIF binding sites by Khamaisi et al. (2015). It has also been shown that endothelin-1 production is controlled by TIMAP-protein phosphatase 1-complex via ECE1 dephosphorylation (Boratko et al. 2016). 
Glioblastoma multiforme is a highly aggressive brain tumor with an exceptionally poor patient outcome despite aggressive therapy including surgery, radiation, and chemotherapy (Yang et al. 2012; Womeldorff et al. 2014; Nayak and Reardon 2017). This aggressive phenotype may be associated with intratumoral hypoxia, which probably plays a key role in glioblastoma growth, development, and angiogenesis. The rapid growth of solid tumors generates microenvironmental changes in regards to hypoxia, nutrient deprivation and acidosis thus inducing the formation of new blood vessels, cell proliferation and survival, processes relying on activation of endoplasmic reticulum stress signaling pathways, which are important to glioma development and a more aggressive behavior through regulation of the cell cycle at distinct stages (Chesney et al. 2014; Yalcin et al. 2014; Zhao et al. 2017). It has been shown that hypoxia-inducible factor-la (HIF1a) blocks differentiation of malignant gliomas possibly through a differentiation defect in malignant gliomas. HIF-1 $\alpha$ is associated with numerous upstream and downstream proteins and an examination of upstream hypoxic and nonhypoxic regulation of HIF1 as well as of downstream HIF1-regulated proteins may provide further insight into the role of this transcription factor in the pathophysiology of glioblastoma and could provide future treatment options for the care of patients with these tumors (Womeldorff et al. 2014; Semenza 2017). It is interesting to note that hypoxia in tumors suppresses cell death pathways, strongly activates glycolysis through variable mechanisms and specifically modulates glutamine metabolism through HIF1 and SIAH2 supports lipid synthesis that is necessary for tumor growth (Sun and Denko 2014). Furthermore, a better knowledge of tumor responses to hypoxia is required to elaborate therapeutical strategies of cell sensibilization, based on the blockade of survival mechanisms (Iurlaro et al. 2017; Semenza 2017; Teramoto and Katoh 2019).

The growing tumor requires the endoplasmic reticulum stress for own neovascularization and growth, for apoptosis inhibition because it has an important position as a signal integrator in both normal and malignant cells (Drogat et al. 2007; Auf et al. 2010; Doultsinos et al. 2017). The endoplasmic reticulum stress signaling pathways have connections with other plasma membrane receptor signaling networks and with numerous metabolic pathways (Bravo et al. 2013; Minchenko et al. 2013; Chevet et al. 2015). Malignant tumors use endoplasmic reticulum stress response and its signaling pathways to adapt and to enhance tumor cells proliferation under stressful environmental conditions (Manie et al. 2014; Papaioannou and Chevet 2018). It is well known that activation of IRE1/ERN1 (inositol requiring enzyme 1/endoplasmic reticulum to nucleus signaling 1) branch of the endoplasmic reticulum stress response is tightly linked to apoptosis and to cell death, and suppression of its function has been demonstrated to result in significant anti-proliferative effect in glioma growth (Auf et al. 2010, 2013; Minchenko et al. 2014, 2015a). Furthermore, inhibition of ERN1endoribonuclease has more strong anti-proliferative effect on glioma cells and other effect on the expression of some ERN1-related pathway genes (Auf et al. 2013; Minchenko et al. 2015c).

It is interesting to note that endoplasmic reticulum stress and hypoxia are two very important and complementary tumor growth factors, and ERN1 mediated stress signaling can significantly modify the effects of hypoxia on gene expressions (Minchenko et al. 2014, 2015a,b, 2016, 2017; Tsymbal et al. 2017). However, the detailed molecular mechanisms of the iteration of hypoxia with ERN1 mediated stress signaling pathway are complex yet and warrant further study.

The aim of this study was to examine the effect of hypoxia on the expression of genes encoded EDN1, its receptors (EDNRA and EDNRB) and ECE1 in U87 glioma cells in response to complete inhibition of ERN1 activity (protein kinase and endoribonuclease) or only its endoribonuclease for evaluation of their possible significance in the control of glioma growth through endoplasmic reticulum stress signaling mediated by IRE1 and hypoxia.

\section{Materials and methods}

Cell lines and culture conditions. The glioma cell line U87 was obtained from ATCC (USA) and grown in high glucose ( $4.5 \mathrm{~g} / \mathrm{l})$ Dulbecco's modified Eagle's minimum essential medium (Gibco, Invitrogen, Carlsbad, CA, USA) supplemented with glutamine ( $2 \mathrm{mM}$ ), 10\% fetal bovine serum (Equitech-Bio, Inc., USA), penicillin (100 units/ml; Gibco) and streptomycin $\left(0.1 \mathrm{mg} / \mathrm{ml}\right.$; Gibco) at $37^{\circ} \mathrm{C}$ in incubator with $5 \% \mathrm{CO}_{2}$. In this work we used three sublines of these cells, which were described previously (Auf et al. 2010, 2013; Minchenko et al. 2015c). One subline was obtained by selection of stable transfected clones with overexpression of vector pcDNA3.1, which was used for creation of dnERN1 and dnrERN1. This untreated subline of glioma cells (control glioma cells) was used as control 1 in the study of the effect of hypoxia 
deprivation on the expression level of genes encoding EDN1 and related proteins (EDNRA, EDNRB, and ECE1). Second subline was obtained by selection of stable transfected clones with overexpression of ERN1 dominant/negative construct (dnERN1), having suppression of both the protein kinase and endoribonuclease activities of this signaling enzyme (Auf et al. 2010). The third sub-line was obtained by the selection of stable transfected clones with the overexpression of dominant-negative ERN1 endoribonuclease mutant (dnrERN1), which was obtained by truncation of the carboxy-terminal 78 amino acids of ERN1 (Auf et al. 2013; Minchenko et al. 2015c). It has been shown that these cells have a low proliferation rate, do not express spliced XBP1, a key transcription factor in ERN1 signaling, and have not the phosphorylated isoform ERN1 after induction of endoplasmic reticulum stress by tunicamycin (Auf et al. 2013; Minchenko et al. 2015c). The expression of the studied genes in cells with a deficiency of ERN1, introduced by dnERN1 and dnrERN1, was compared with cells transfected with the previously mentioned, empty vector (control glioma cells, pcDNA3.1). All used in this study sublines of glioma cells are grown in the presence of geneticin (G418) while these cells carrying empty vector pcDNA3.1, dnERN1 or dnrERN1 constructs. Hypoxic condition was created in special incubator with 3\% oxygen and 5\% carbon dioxide levels; culture plates with complete DMEM were exposed to these conditions for $16 \mathrm{~h}$.

RNA isolation. Total RNA was extracted from glioma cells using the Trizol reagent according to manufacturer's protocol (Invitrogen, Carlsbad, CA, USA). The RNA pellets were washed with $75 \%$ ethanol and dissolved in nuclease-free water. For additional purification RNA samples were re-precipitated with 95\% ethanol and re-dissolved again in nuclease-free water. RNA concentration and spectral characteristics were measured using NanoDrop Spectrophotometer ND1000 (PEQLAB, Biotechnologie GmbH).

Reverse transcription and quantitative PCR analysis. The effect of hypoxia on the expression levels of endothelin-1 and its receptors (EDNRA and EDNRB) mRNAs as well as ECE1 and ACTB mRNAs were measured in control U87 glioma cells and cells with a deficiency of ERN1, introduced by dnERN1 and dnrERN1, by quantitative polymerase chain reaction using SYBRGreen Mix (ABgene, Thermo Fisher Scientific, Epsom, Surrey, UK) and qPCR „RotorGene RG-3000” (Corbett Research, Germany) and "QuantStudio 5 Real-Time PCR System" (Applied Biosystems, USA). Thermo Scientific Verso cDNA Synthesis Kit (Germany) was used for reverse transcription as described previously (Minchenko et al. 2019). Polymerase chain reaction was performed in triplicate. The expression of beta-actin mRNA was used as control of analyzed RNA quantity. The pair of primers specific for each studied gene was received from Sigma-Aldrich (St. Louis, MO, U.S.A.) and used for quantitative polymerase chain reaction (Table 1).

Quantitative PCR analysis was performed using a special computer program "Differential expression calculator" and statistical analysis using Excel program and OriginPro 7.5 software as described previously (Minchenko et al. 2015c). Comparison of two means was performed by the use of two-tailed Student's t-test. A p-value $<0.05$ was considered significant in all cases. The values of EDN1, EDNRA,

Table 1

Characteristics of the primers used for quantitative real-time polymerase chain reaction

\begin{tabular}{|c|c|c|c|c|}
\hline Gene symbol & Gene name & Primer's sequence & $\begin{array}{c}\text { Nucleotide numbers } \\
\text { in sequence }\end{array}$ & $\begin{array}{c}\text { GenBank accession } \\
\text { number }\end{array}$ \\
\hline EDN1 & endothelin 1 & $\begin{array}{l}\text { F: 5'-tctctctgctgtttgtggct } \\
\text { R: 5'-ccaggtggcagaagtagaca }\end{array}$ & $\begin{array}{l}292-311 \\
477-458\end{array}$ & NM_001955 \\
\hline EDNRA & endothelin receptor type A & $\begin{array}{l}\text { F: } 5 \text { '-tcgtgttcagggaattggga } \\
\text { R: } 5 \text {-atagaacccgaagagccacc }\end{array}$ & $\begin{array}{c}923-942 \\
1139-1120\end{array}$ & NM_001957 \\
\hline EDNRB & endothelin receptor type B & $\begin{array}{l}\text { F: 5'-gaacaagtgcatgcgaaacg } \\
\text { R: } 5 \text {-actcagcacagtgattccca }\end{array}$ & $\begin{array}{c}812-831 \\
1001-982\end{array}$ & NM_000115 \\
\hline ECE1 & endothelin converting enzyme 1 & $\begin{array}{l}\text { F: 5'-tgcggaaaacaagctccttc } \\
\text { R: 5'-gatctcggtggctatgctct }\end{array}$ & $\begin{array}{l}1245-1264 \\
1450-1431\end{array}$ & NM_001397 \\
\hline АСТВ & beta-actin & $\begin{array}{l}\text { F: 5'-ggacttcgagcaagagatgg } \\
\text { R: 5'-agcactgtgttggcgtacag }\end{array}$ & $\begin{array}{l}747-766 \\
980-961\end{array}$ & NM_001101 \\
\hline MiRNA19A & microRNA 19a-3p & F: 5'-tgtgcaaatccatgcaaaactga & $1-23$ & MIMAT0000073 \\
\hline MiRNA96 & microRNA 96 & F: 5'-tttggcactagcacatttttgct & $1-23$ & MIMAT0000095 \\
\hline MiRNA206 & microRNA 206 & F: 5'-tggaatgtaaggaagtgtgtgg & $1-22$ & MIMAT0000462 \\
\hline
\end{tabular}


$E D N R B$, and ECE1 gene expressions were normalized to the expression of beta-actin mRNA and represent as percent of control (100\%). All values are expressed as mean \pm SEM from triplicate measurements performed in 4 independent experiments. The amplified DNA fragments were also analyzed on a $2 \%$ agarose gel and that visualized by $\mathrm{SYBR}^{\star}$ Safe DNA Gel Stain (Life Technologies, Carlsbad, CA, USA).

\section{Results}

To investigate a possible role of endoplasmic reticulum stress signaling mediated by ERN1 bifunctional enzyme in the expression level of mRNA for endothelin-1 receptor and its cognate receptors (EDNRA and EDNRB) as well as endothelin converting enzyme 1 we studied in U87 glioma cells with a deficiency of ERN1: full deficiency, introduced by dnERN1 (cells without both protein kinase and endoribonuclease activities), and with deficiency in ERN1 endoribonuclease, introduced by dnrERN1 (cells without endoribonuclease activity only). As shown in Figure 1, the expression of EDN1 mRNA in U87 glioma cells, transfected by dnERN1, is strongly

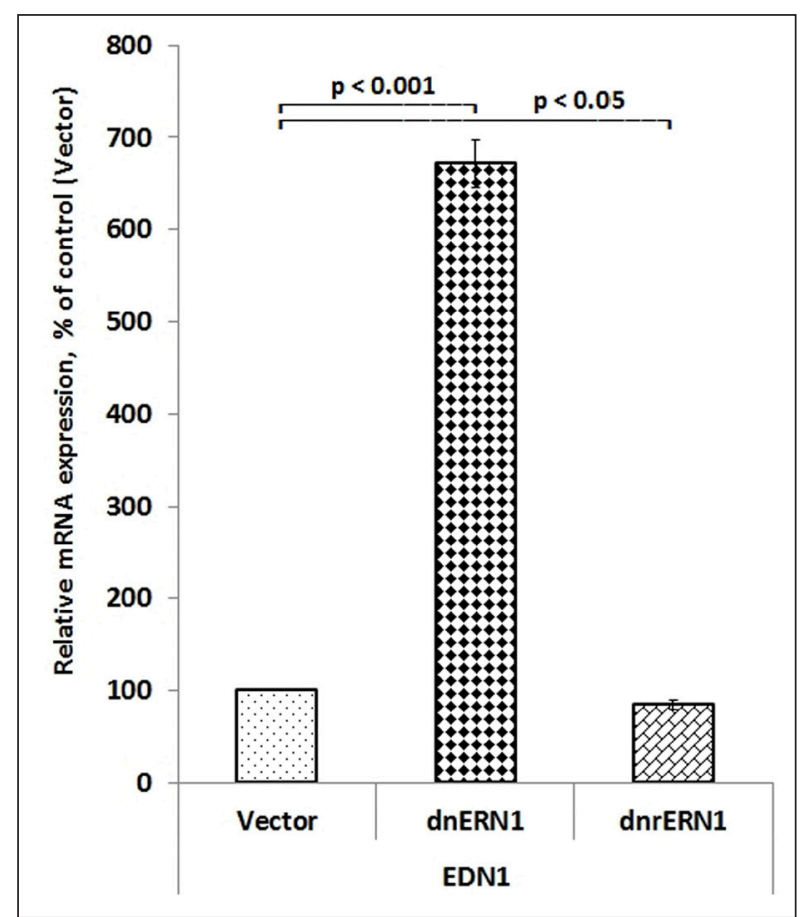

Figure 1. Endothelin 1 (EDN1) gene expression level in control U87 glioma cells (Vector) and cells with a blockade of the ERN1 by dnERN1 and with a blockade of the ERN1 endoribonuclease by dnrERN1 measured by qPCR. Values of EDN1 mRNA expression were normalized to beta-actin mRNA level and represented as percent for control $1(100 \%) ; n=4$. up-regulated $(+572 \%)$ in comparison with control glioma cells, transfected by empty vector. At the same time, inhibition of ERN1 endoribonuclease has small but statistically significant effect $(-15 \%)$ on endothelin-1 gene expression (Figure 1). We next investigated the effect of ERN1 inhibition on the expression of gene encoding endothelin receptor type A in glioma cells. As shown in Figure 2, the expression of EDNRA mRNA is strongly up-regulated (+119\%) in U87 glioma cells without both protein kinase and endoribonuclease activities in comparison with cells, transfected by empty vector pcDNA3.1. Furthermore, similar results in the expression of EDNRA gene we received in glioma cells with deficiency in ERN1 endoribonuclease, introduced by dnrERN1 (+94\%) as compared to control glioma cells (Figure 2).

We also studied the expression of gene encoding endothelin receptor type B in glioma cells in relation to suppression of functional activity of ERN1 signaling enzyme. As also shown in Figure 3, the expression level of EDNRB mRNA is also up-regulated in glioma cells transfected by dnERN1 but the effect was much smaller in comparison with cells, transfected by empty vector (+35\%). Moreover, inhi-

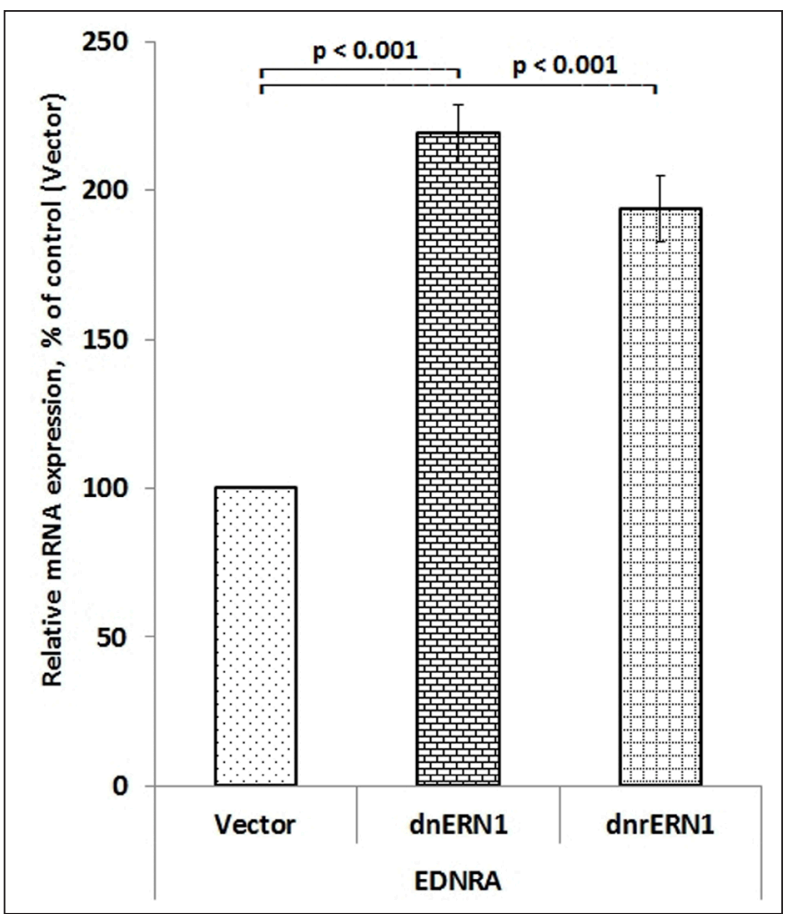

Figure 2. Endothelin receptor type A (EDNRA) gene expression level in control U87 glioma cells (Vector) and cells with a blockade of the ERN1 by dnERN1 and with a blockade of the ERN1 endoribonuclease by dnrERN1 measured by qPCR. Values of EDNRA mRNA expression were normalized to beta-actin mRNA level and represented as percent for control 1 (100\%); $\mathrm{n}=4$. 
bition of endoribonuclease activity of ERN1 signaling enzyme leads to down-regulation of EDNRB gene expression $(-35 \%)$ as compared to control glioma cells (Figure 3 ).

Investigation of gene encoding endothelin converting enzyme 1, which play an important role in the regulation of endothelin-1 activity, showed its strong sensitivity to the suppression of ERN1 signaling enzyme function (Figure 4). Thus, the expression level of ECE1 gene is significantly up-regulated $(+143 \%)$ in glioma cells without both protein kinase and endoribonuclease activities in comparison with cells, transfected by empty vector. Very similar results were observed in glioma cells without endoribonuclease activity of ERN1 signaling enzyme $(+154 \%)$ as comparted to control cells (Figure 4). Thus, inhibition of both enzymatic activities of ERN1 signaling enzyme and only endoribonuclease has similar effect on the expression of EDNRA and ECE1 genes and different, opposite, effect on EDN1 and EDNRB genes.

We next investigated the effect of hypoxia on the expression of gene encoding endothelin-1 in glioma

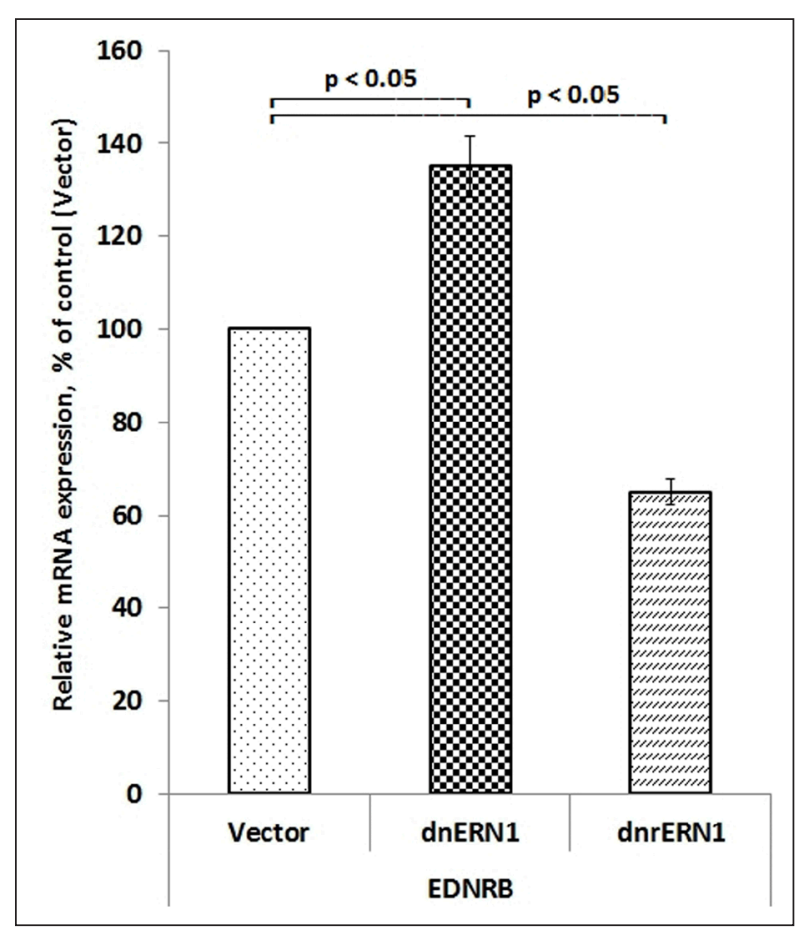

Figure 3. Endothelin receptor type B (EDNRB) gene expression level in control U87 glioma cells (Vector) and cells with a blockade of the ERN1 by dnERN1 and with a blockade of the ERN1 endoribonuclease by dnrERN1 measured by qPCR. Values of EDNRB mRNA expression were normalized to beta-actin mRNA level and represent as percent for control 1 $(100 \%) ; n=4$. cells in relation to complete inhibition of ERN1 signaling enzyme function (both protein kinase and endoribonuclease activities). It was shown that in control glioma cells (transfected by empty vector) the expression level of EDN1 gene is up-regulated $(+251 \%)$ under hypoxic condition in comparison with cells growing in regular condition (Figure 5). At the same time, inhibition of ERN1 signaling enzyme function significantly suppresses the effect of hypoxia on endothelin-1 gene expression as compared to corresponding control cells, transfected by dnERN1 (+60\%; Figure 5). Furthermore, effect of hypoxia on the expression of endothelin receptor type $\mathrm{A}$ and $\mathrm{B}$ genes in glioma cells in control glioma cells was different (Figure 6 and Figure 7). Thus, exposure cells under hypoxia leads to up-regulation of EDNRA $(+44 \%)$ and down-regulation of EDNRB (-21\%) genes. It is interesting to note that complete inhibition of ERN1 signaling enzyme function (both protein kinase and endoribonuclease activities) modifies effect of hypoxia on these endothelin receptor genes. Thus, the expression of endothelin receptor type A is down-regulated (-51\%) and endothelin receptor type

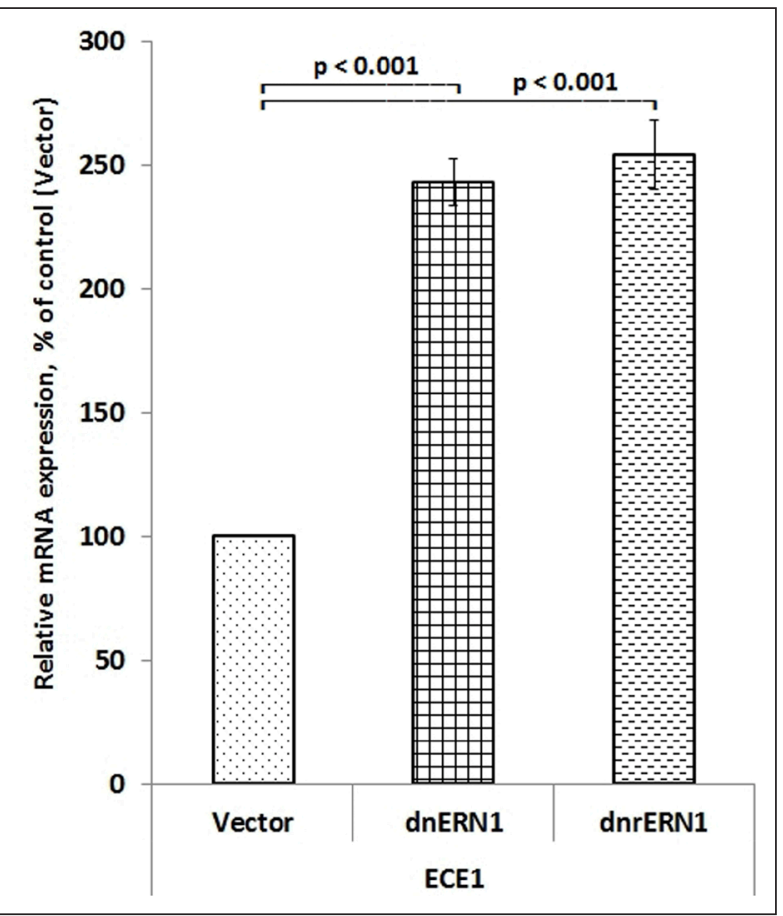

Figure 4. Endothelin converting enzyme 1 (ECE1) gene expression level in control U87 glioma cells (Vector) and cells with a blockade of the ERN1 by dnERN1 and with a blockade of the ERN1 endoribonuclease by dnrERN1 measured by qPCR. Values of ECE1 mRNA expression were normalized to beta-actin mRNA level and represented as percent for control $1(100 \%) ; n=4$. 


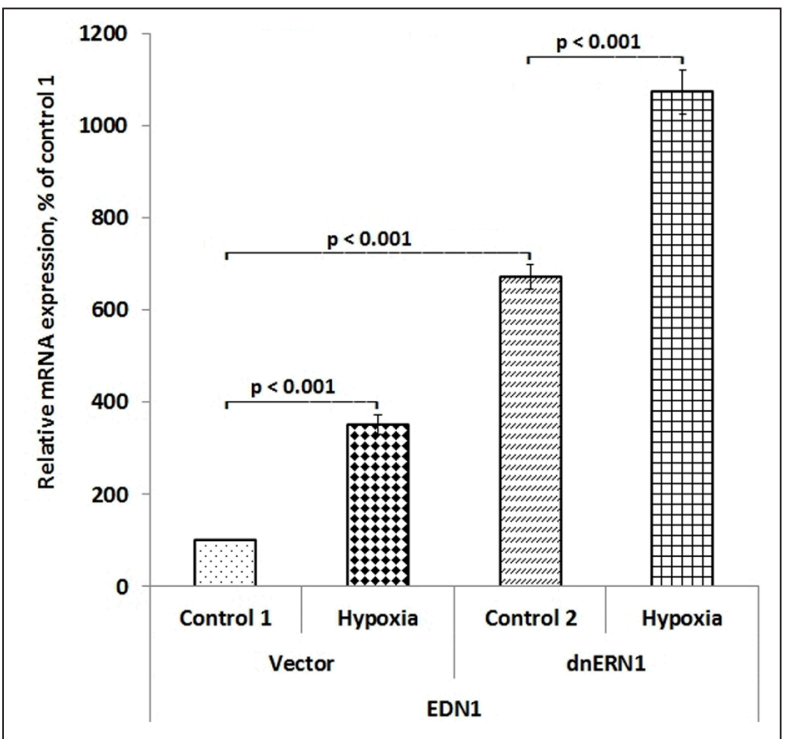

Figure 5. Effect of hypoxia on the expression level of EDN1 mRNA in control U87 glioma cells (Vector) and cells with a blockade of the ERN1 by dnERN1 measured by qPCR. Values of EDN1 mRNA expression were normalized to beta-actin mRNA level and represented as percent for control $1(100 \%) ; n=4$.

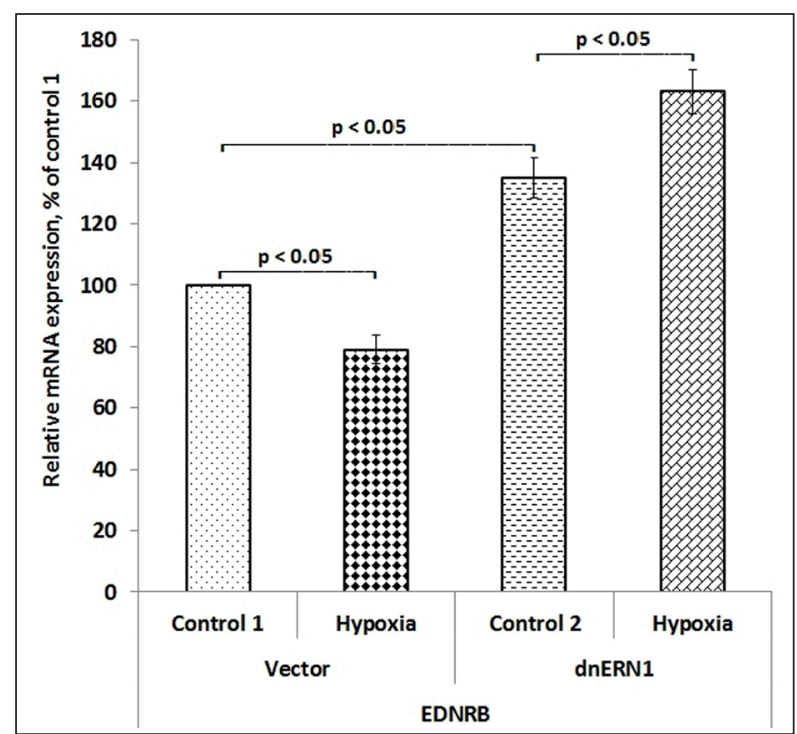

Figure 7. Effect of hypoxia on the expression level of EDNRB mRNA in control U87 glioma cells (Vector) and cells with a blockade of the ERN1 by dnERN1 measured by qPCR. Values of EDNRB mRNA expression were normalized to beta-actin mRNA level and represented as percent for control $1(100 \%) ; n=4$.

$\mathrm{B}$ is up-regulated $(+21 \%)$ in comparison to control glioma cells, transfected with dnERN1 (Figure 6 and Figure 7). As shown in Figure 8, exposure glioma cells under hypoxic condition leads to significant up-regulation of endothelin converting enzyme 1

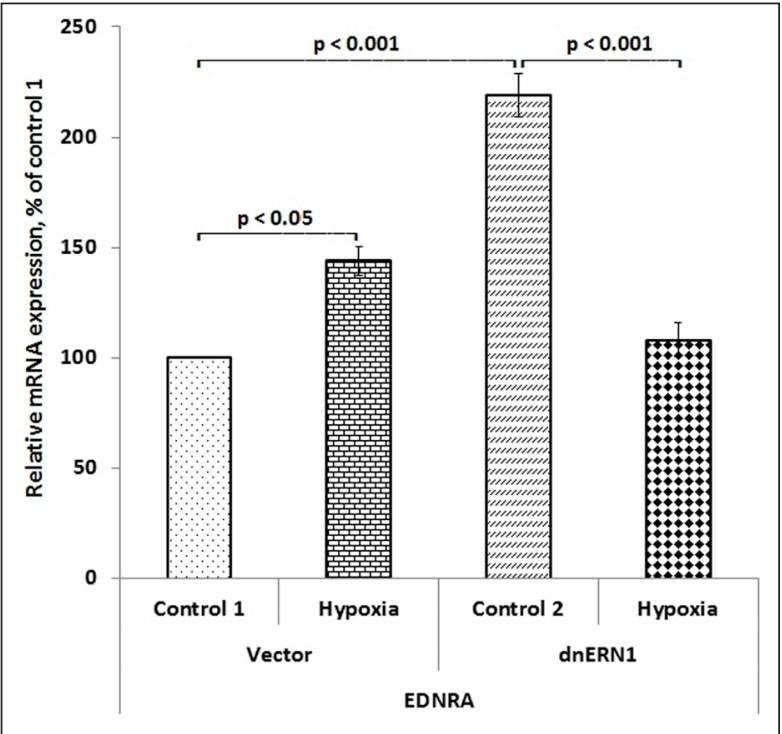

Figure 6. Effect of hypoxia on the expression level of EDNRA mRNA in control U87 glioma cells (Vector) and cells with a blockade of the ERN1 by dnERN1 measured by qPCR. Values of this mRNA expression were normalized to beta-actin mRNA level and represented as percent for control $1(100 \%) ; n=4$.

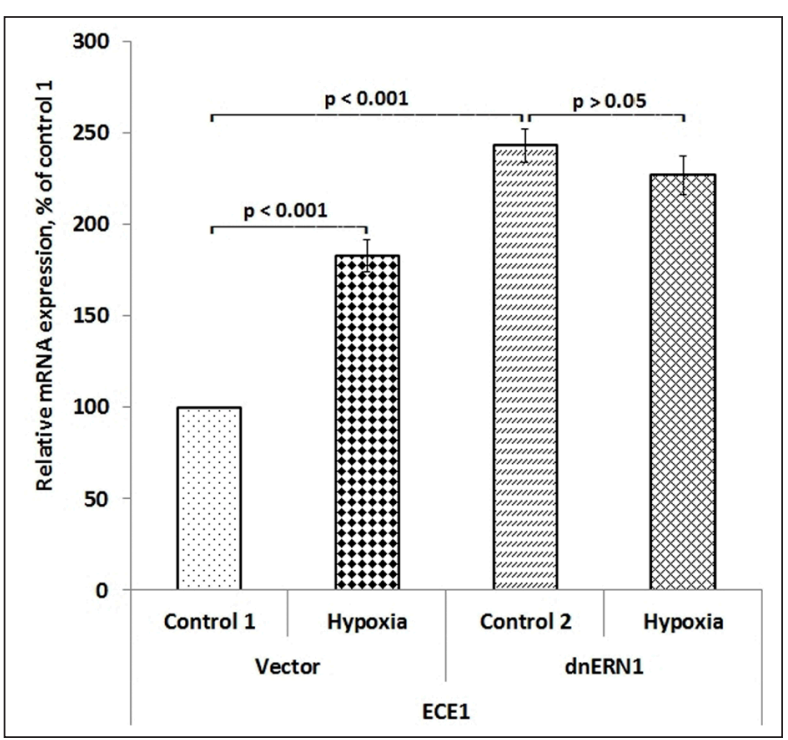

Figure 8. Effect of hypoxia on the expression level of ECE1 mRNA in control U87 glioma cells (Vector) and cells with a blockade of the ERN1 by dnERN1 measured by qPCR. Values of this mRNA expression were normalized to beta-actin mRNA level and represented as percent for control $1(100 \%) ; n=4$.

gene expression $(+83 \%)$ in comparison with control cells growing under normoxic condition. At the same time, inhibition of both enzymatic activities of ERN1 completely eliminates effect of hypoxia on ECE1 gene expression (Figure 8). 
Thus, hypoxia up-regulates the expression of all studied genes except EDNRB gene and inhibition of endoplasmic reticulum stress signaling mediated by ERN1 modifies the effect of hypoxia on the expression of endothelin-1, its receptors and endothelin converting enzyme 1 in gene-specific manner.

We also studied the expression level of microRNA miR-206, miR-96, and miR-19a, which have specific binding sites in mRNA EDN1, EDNRA, and EDNRB, correspondingly, and can participate in posttranscriptional regulation of these mRNA expressions. As shown in Figure 9, the expression level of microRNA miR-206, miR-96, and miR-19a is down-regulated, being more significant for miR-96 and miR-19a, in glioma cells without both ERN1 protein kinase and endoribonuclease activities (full ERN1 deficiency, introduced by dnERN1) in comparison with control cells. Therefore, the down-regulation of these microRNAs correlates with up-regulation of corresponding mRNA (EDN1, EDNRA, and EDNRB) in ERN1 knockdown glioma cells.

\section{Discussion}

In this work, we studied the expression of genes encoding endothelin-1, its receptors (EDNRA and EDNRB) and endothelin converting enzyme 1, which have relation to functional activity of EDN1, in relation to inhibition of ERN1, the major signaling pathway of the unfolded protein response, in U87 glioma cells with full ERN1deficiency, introduced by dnERN1 (cells without both protein kinase and endoribonuclease activities), and with deficiency in ERN1 endoribonuclease, introduced by dnrERN1 (cells without endoribonuclease activity only). It is important for evaluation of possible significance of these genes in the control of glioma growth through endoplasmic reticulum stress signaling mediated by ERN1 because this stress signaling pathway is involved in numerous metabolic pathways and inhibition of ERN1 signaling enzyme activity in glioma cells had clear anti-tumor effects (Auf et al. 2010, 2013; Bravo et al. 2013; Manie et al. 2014; Minchenko et al. 2013, 2014, 2015a,c; Logue et al. 2018).

We have shown that the expression of all studied genes $E D N 1, E D N R A, E D N R B$, and ECE1 is responsible to endoplasmic reticulum stress signaling mediated by ERN1 and up-regulated in glioma cells without both protein kinase and endoribonuclease activities. However, molecular mechanisms of these changes are different, because inhibition of ERN1 endoribonuclease activity leads to up-regulation of $E D N R A$ and ECE1 genes only. Thus, the expression

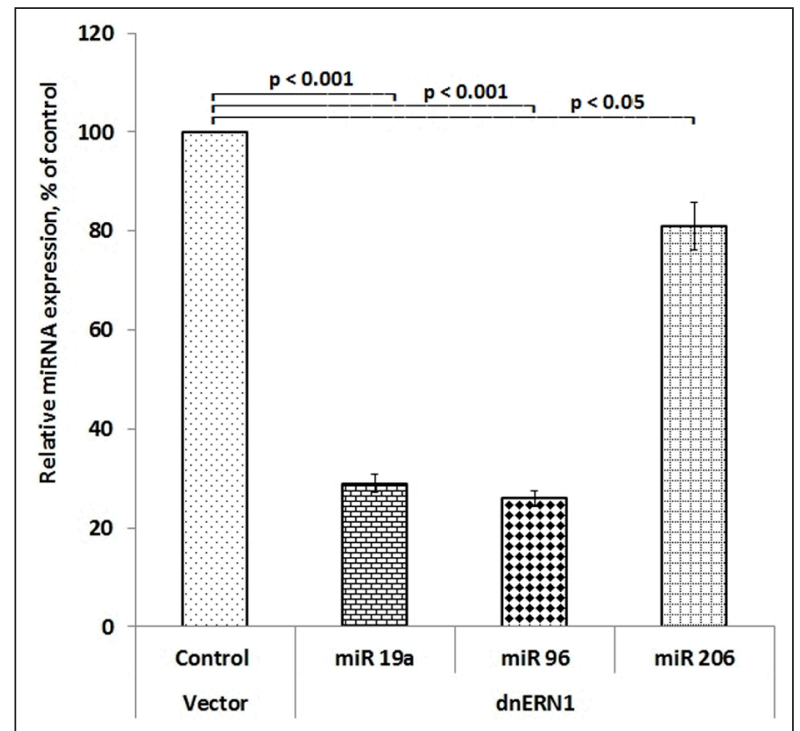

Figure 9. Expression level of microRNA miR 19a, miR 96, and miR 206 in control U87 glioma cells (Vector) and cells with a blockade of the ERN1 by dnERN1 measured by qPCR. Values of these mRNA expressions were normalized to beta-actin mRNA level and represented as percent for control $1(100 \%) ; n=4$.

of EDNRA and ECE1 genes is regulated by ERN1 endoribonuclease, but EDN1 and EDNRB genes preferentially by ERN1 protein kinase by mechanisms described by Auf et al. (2013) and Minchenko et al. (2015c). Functional significance of up-regulation EDN1 and its cognate receptors (EDNRA and EDNRB) as well as endothelin converting enzyme 1 under inhibition of both ERN1 protein kinase and endoribonuclease activities because there is data that endothelin system is involved in cancer biology as well as in many other important processes and that expression of EDN1, EDNRA, EDNRB, and ECE1 genes is elevated in several tumors and cancer cell lines (Teoh et al. 2014; Cook et al. 2015; Niechi et al. 2015; Tapia and Niechi 2019).

At the same time, showed that the expression of all studied EDNland EDNRB genes is regulated preferentially by ERN1 protein kinase and that inhibition of ERN1 endoribonuclease activity leads to downregulation of $E D N R B$ and $E D N 1$ genes in glioma cells. These results correlate well with a data Auf et al. (2013) and Minchenko et al. (2015c) that inhibition of the endoribonuclease activity of ERN1 alone leads to a more robust suppression of malignant cell proliferation as compared to cells lacking both kinase and endoribonuclease activity. It is possible that endothelin system is involved in various very important processes; not only in cancer development, but the prominent pathophysiological aspects of the endo- 


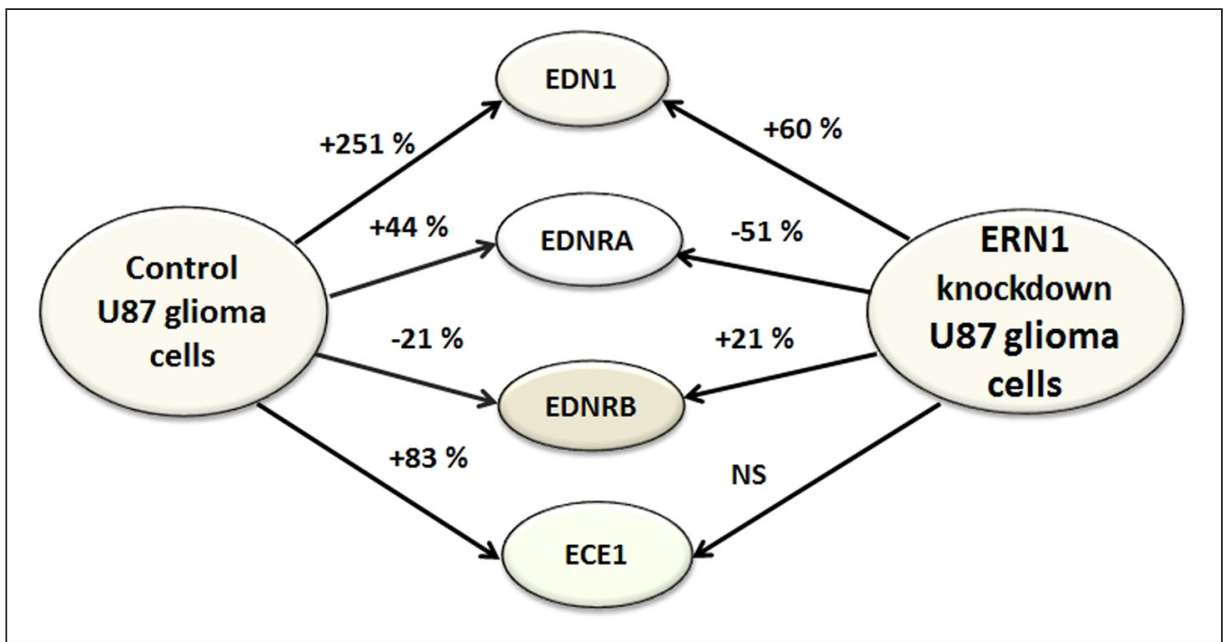

Figure 10. Schematic demonstration of EDN1, EDNRA, EDNRB, and ECE1 genes expression profile in control and ERN1 knockdown glioma cells under hypoxia; NS - no significant changes.

thelin system in glioma cells as well as in astrocytes is not clear yet.

We also showed that hypoxia enhanced the expression of EDN1, EDNRA, and ECE1 genes and that knockdown of ERN1 signaling enzyme function significantly modified the response of all studied genes of endothelin system to hypoxia (Figure 10). Previously we have shown that hypoxia up-regulates HIF1A protein both in control and ERN1 knockdown glioma cells, but inhibition of ERN1 signaling enzyme decreases this protein level without significant changes in sensitivity to hypoxia (Tsymbal et al. 2017). Furthermore, hypoxia stimulates human preproendothelin-1 promoter activity in transgenic mice and in microvascular endothelial cells through interaction of transcription factor HIF with specific hypoxia responsible element in EDN1 promoter (Aversa et al. 1997; Minchenko and Caro 2000). However, hypoxic regulation of the EDN1 gene expression is associated with protein kinases and functional activity of other transcriptional factors as well as with signaling pathways of endoplasmic reticulum stress (Minchenko and Caro 2000; Stow et al. 2011; Minchenko et al. 2015b, 2017; Tsymbal et al. 2017; Zhang et al. 2018).

It is possible that hypoxic stimulation of ENDRA and ECE1 gene expressions in glioma cells is also realized by transcription factor HIF through specific hypoxia responsible elements, which are present in promoter region of these genes. Khamaisi et al. (2015) have shown that hypoxia increases the expression level of ECE1 in vascular endothelial cells through hypoxia responsible elements, identified in promoter and intron regions. Furthermore, in promoter region of ENDRA gene we identified two potential binding sites for HIF and three - for XBP1, a major transcription factor of ERN1 signaling, and one of them overlapping with HIF (CCACGCG). It is possible that hypoxic regulation of ENDRA gene is realized through interaction HIF and XBP1 transcription factors on such binding sites, at least partially, and elimination of active XBP1 by ERN1 knockdown (Auf et al. 2010) reduces effect of hypoxia on ENDRA gene expression. At the same time, many factors can facilitate interaction of HIF with endoplasmic reticulum stress response-signaling pathway mediated by ERN1 (Manie et al. 2014; Sun and Denko 2014; Chevet et al. 2015). There are data that HIF-1a is associated with numerous upstream and downstream proteins and an examination of upstream hypoxic and nonhypoxic regulation of HIF1 as well as of downstream HIF1-regulated proteins may provide further insight into the role of this transcription factor in the pathophysiology of glioblastoma at the molecular level (Womeldorff et al. 2014; Semenza 2017).

Our data showed that hypoxia down-regulates ENDRB gene expression and that inhibition of ERN1 signaling leads to up-regulation of this gene. These results conform to data that intrarenal activation of endothelin type B receptors improve kidney oxygenation in type 1 diabetic rats and that pharmacological inhibition and cardiac-specific knockout of endothelin receptor type B lead to cardiac resistance to extreme hypoxia (Franzen et al. 2018; Stobdan et al. 2018).

It is possible that hypoxic induction of EDN1, $E D N R A$, and ECE1 gene expressions reflects real pro-tumor effects of hypoxia and that anti-proli- 
ferative effect of ERN1 knockdown (Auf et al. 2010, 2013; Minchenko et al. 2015c) agree well with strong reduction of the sensitivity of EDN1 and ECE1 gene expressions to hypoxia as well as with down-regulation of the expression of EDNRA gene under hypoxic condition (Figure 10). These results validate tight interaction of endoplasmic reticulum stress signaling pathways with hypoxic regulation of the expression of genes encoding endothelin-1, its receptor type A, and endothelin converting enzyme 1 , which have relation to functional activity of EDN1, but precise molecular mechanisms is not clear yet and warrant further investigations.

We also observed down-regulation of microRNA miR-206, miR-96, and miR-19a, which have specific binding sites in mRNA EDN1, EDNRA, and EDNRB, correspondingly, in glioma cells with ERN1 knockdown. Furthermore, the level of these mRNA is upregulated. Our findings provide evidence that the enhanced expression pattern of EDN1, EDNRA, and EDNRB mRNAs is regulated both at transcriptional and post-transcriptional levels. Furthermore, downregulation of microRNA miR-206, miR-96, and miR-19a agree well with their role in tumorigenesis (Lin et al. 2010; Liang et al. 2011; Singh et al. 2013; Chua and Adams 2017). These results conform to data that functions of noncoding RNA in regulating adaptive pathways in both physiological and pathophysiological conditions, illustrating how they operate within the known UPR functions and contribute to diverse cellular outcomes (McMahon et al. 2017).

This study provides unique insights into the molecular mechanisms regulating the expression of genes encoding endothelin-1, its receptors and endothelin converting enzyme 1 in glioma cells in response to complete inhibition of ERN1 activity (protein kinase and endoribonuclease) or only its endoribonuclease and their correlation with reduced cell proliferation in cells harboring dnERN1, attesting to the fact that endoplasmic reticulum stress is a necessary component of malignant tumor growth and cell survival. Moreover, we identified microRNA, which down-regulated expression can contribute to posttranscriptional up-regulation of $E D N 1, E D N R A$, and EDNRB mRNAs in ERN1 knockdown glioma cells. Furthermore, our results demonstrate that hypoxic induction of EDN1, EDNRA, and ECE1 gene expressions in control glioma cells reflects real pro-tumor effects of hypoxia and that anti-proliferative effect of ERN1 knockdown is associated with strong reduction of the sensitivity of EDN1 and ECE1 gene expressions to hypoxia as well as with down-regulation of the expression of EDNRA gene under hypoxic condition. It is interesting to note that our results validate tight interaction of endoplasmic reticulum stress signaling pathways with hypoxic regulation of the expression of genes encoding endothelin-1, its receptor type $A$, and endothelin converting enzyme 1, which have relation to functional activity of EDN1, but the detailed molecular mechanisms of this regulation have not been yet clearly defined and warrant further investigation.

\section{Acknowledgement}

This work was funded by the State Budget Program "Support for the Development of Priority Areas of Scientific Research" (Code: 6541230).

\section{References}

Arabanian LS, Johansson P, Staffas A, Nilsson T, Rouhi A, Fogelstrand L, Palmqvist L. The endothelin receptor type A is a downstream target of Hoxa9 and Meis1 in acute myeloid leukemia. Leuk Res 75, 61-68, 2018.

AufG, Jabouille A, Guerit S, Pineau R, Delugin M, Bouchecareilh M, Favereaux A, Maitre M, Gaiser T, von Deimling A, Czabanka M, Vajkoczy P, Chevet E, Bikfalvi A, Moenner M. A shift from an angiogenic to invasive phenotype induced in malignant glioma by inhibition of the unfolded protein response sensor IRE1. Proc Natl Acad Sci U S A 107, 15553-15558, 2010.

Auf G, Jabouille A, Delugin M, Guerit S, Pineau R, North S, Platonova N, Maitre M, Favereaux A, Vajkoczy P, Seno M, Bikfalvi A, Minchenko D, Minchenko O, Moenner M. High epiregulin expression in human U87 glioma cells relies on IRE1alpha and promotes autocrine growth through EGF receptor. BMC Cancer 13, 597, 2013.

Aydin AF, Vural P, Dogru-Abbasoglu S, Cil E. The endothelin 1 and endothelin receptor A gene polymorphisms increase the risk of developing papillary thyroid cancer. Mol Biol Rep 46, 199-205, 2019.

Aversa CR, Oparil S, Caro J, Li H, Sun S-D, Chen Y-F, Swerdel MR, Monticello TM, Durham SK, Minchenko A, Lira SA, Webb ML. Hypoxia stimulates human preproendothelin-1 promoter activity in transgenic mice. Amer J Physiol 273, L848-L855, 1997. 
Bao N, Fang B, Lv H, Jiang Y, Chen F, Wang Z, Ma H. Upregulation of miR-199a-5p protects spinal cord against ischemia/reperfusion-induced injury via downregulation of ECE1 in rat. Cell Mol Neurobiol 38, 1293-1303, 2018.

Boratko A, Vereb Z, Petrovski G, Csortos C. TIMAP-protein phosphatase 1-complex controls endothelin-1 production via ECE-1 dephosphorylation. Int J Biochem Cell Biol 73, 11-18, 2016.

Bravo R, Parra V, Gatica D, Rodriguez AE, Torrealba N, Paredes F, Wang ZV, Zorzano A, Hill JA, Jaimovich E, Quest AF, Lavandero S. Endoplasmic reticulum and the unfolded protein response: dynamics and metabolic integration. Int Rev Cell Mol Biol 301, 215-290, 2013.

Chesney J, Clark J, Klarer AC, Imbert-Fernandez Y, Lane AN, Telang S. Fructose-2,6-bisphosphate synthesis by 6-phosphofructo-2-kinase/fructose-2,6-bisphosphatase 4 (PFKFB4) is required for the glycolytic response to hypoxia and tumor growth. Oncotarget 5, 6670-6686, 2014.

Chevet E, Hetz C, Samali A. Endoplasmic reticulum stress-activated cell reprogramming in oncogenesis. Cancer Discov 5, 586-597, 2015.

Chua FY, Adams BD. Androgen receptor and miR-206 regulation in prostate cancer. Transcription 8, 313-327, 2017.

Cook N, Brais R, Qian W, Hak CC, Corrie PG. Endothelin-1 and endothelin B receptor expression in pancreatic adenocarcinoma. J Clin Pathol 68, 309-313, 2015.

Dashwood MR, Loesch A. Endothelin-1 as a neuropeptide: neurotransmitter or neurovascular effects? J Cell Commun Signal 4, 51-62, 2009.

Dojo Soeandy C, Salmasi F, Latif M, Elia AJ, Suo NJ, Henderson JT. Endothelin-1-mediated cerebral ischemia in mice: early cellular events and the role of caspase-3. Apoptosis 24, 578-595, 2019.

Doultsinos D, Avril T, Lhomond S, Dejeans N, Guedat P, Chevet E. Control of the unfolded protein response in health and disease. SLAS Discov 22, 787-800, 2017.

Drogat B, Auguste P, Nguyen DT, Bouchecareilh M, Pineau R, Nalbantoglu J, Kaufman RJ, Chevet E, Bikfalvi A, Moenner M. IRE1 signaling is essential for ischemia-induced vascular endothelial growth factor-A expression and contributes to angiogenesis and tumor growth in vivo. Cancer Res 67, 6700-6707, 2007.

Farhat FP, Martins CB, De Lima LH, Isoldi MC, Castrucci AM. Melanopsin and clock genes: regulation by light and endothelin in the zebrafish ZEM-2S cell line. Chronobiol Int 26, 1090-1119, 2009.

Fomin V, Richard P, Hoque M, Li C, Gu Z, Fissore-O’Leary M, Tian B, Prives C, Manley JL. The C9ORF72 gene, implicated in amyotrophic lateral sclerosis and frontotemporal dementia, encodes a protein that functions in control of endothelin and glutamate signaling. Mol Cell Biol 38, e00155-18, 2018.

Franzen S, Pihl L, Fasching A, Palm F. Intrarenal activation of endothelin type B receptors improves kidney oxygenation in type 1 diabetic rats. Am J Physiol Renal Physiol 314, F439-F444, 2018.

Iurlaro R, Puschel F, Leon-Annicchiarico CL, O’Connor H, Martin SJ, Palou-Gramon D, Lucendo E, Munoz-Pinedo C. Glucose deprivation induces ATF4-mediated apoptosis through TRAIL death receptors. Mol Cell Biol 37, e00479-16, 2017.

Khamaisi M, Toukan H, Axelrod JH, Rosenberger C, Skarzinski G, Shina A, Meidan R, Koesters R, Rosen S, Walkinshaw G, Mimura I, Nangaku M, Heyman SN. Endothelin-converting enzyme is a plausible target gene for hypoxia-inducible factor. Kidney Int 87, 761-770, 2015.

Khimji AK, Rockey DC. Endothelin-biology and disease. Cell Signal 22, 1615-1625, 2010.

Liang Z, Li Y, Huang K, Wagar N, Shim H. Regulation of miR-19 to breast cancer chemoresistance through targeting PTEN. Pharm Res 28, 3091-3100, 2011.

Lin H, Dai T, Xiong H, Zhao X, Chen X, Yu C, Li J, Wang X, Song L. Unregulated miR-96 induces cell proliferation in human breast cancer by downregulating transcriptional factor FOXO3a. PLoS One 5, e15797, 2010.

Logue SE, McGrath EP, Cleary P, Greene S, Mnich K, Almanza A, Chevet E, Dwyer RM, Oommen A, Legembre P, Godey F, Madden EC, Leuzzi B, Obacz J, Zeng Q, Patterson JB, Jager R, Gorman AM, Samali A. Inhibition of IRE1 RNase activity modulates the tumor cell secretome and enhances response to chemotherapy. Nat Commun 9, 3267, 2018.

Manie SN, Lebeau J, Chevet E. Cellular mechanisms of endoplasmic reticulum stress signaling in health and disease. 3. Orchestrating the unfolded protein response in oncogenesis: an update. Am J Physiol Cell Physiol 307, C901-C907, 2014.

McMahon M, Samali A, Chevet E. Regulation of the unfolded protein response by noncoding RNA. Am J Physiol Cell Physiol 313, C243-C254, 2017.

Minchenko AG, Armstead VE, Opentanova IL, Lefer AM. Endothelin-1, endothelin receptors and ecNOS gene expression in vital organs during traumatic shock in rats. Endothelium 6, 303-314, 1999. 
Minchenko A, Caro J. Regulation of endothelin-1 gene expression in human microvascular endothelial cells by hypoxia and cobalt: role of hypoxia responsible element. Mol Cell Biochem 208, 53-62, 2000.

Minchenko AG, Stevens MJ, White L, Abatan OI, Komjati K, Pacher P, Szabo C, Obrosova IG. Diabetes-induced overexpression of endothelin-1 and endothelin receptors in the rat renal cortex is mediated via poly(ADPribose)polymerase activation. FASEB J 17, 1514-1516, 2003.

Minchenko OH, Kharkova AP, Bakalets TV, Kryvdiuk IV. Endoplasmic reticulum stress, its sensor and signaling systems and the role in regulation of gene expressions at malignant tumor growth and hypoxia. Ukr Biokhim Zh 85, 5-16, 2013.

Minchenko DO, Danilovskyi SV, Kryvdiuk IV, Bakalets TV, Lypova NM, Karbovskyi LL, Minchenko OH. Inhibition of ERN1 modifies the hypoxic regulation of the expression of TP53-related genes in U87 glioma cells. Endoplasm Reticul Stress Dis 1, 18-26, 2014.

Minchenko DO, Kharkova AP, Tsymbal DO, Karbovskyi LL, Minchenko OH. Expression of insulin-like growth factor binding protein genes and its hypoxic regulation in U87 glioma cells depends on ERN1 mediated signaling pathway of endoplasmic reticulum stress. Endocr Regul 49, 73-83, 2015a.

Minchenko OH, Tsymbal DO, Minchenko DO, Kovalevska OV, Karbovskyi LL, Bikfalvi A. Inhibition of ERN1 signaling enzyme affects hypoxic regulation of the expression of E2F8, EPAS1, HOXC6, ATF3, TBX3 and FOXF1 genes in U87 glioma cells. Ukr Biochem J 87, 76-87, $2015 \mathrm{~b}$.

Minchenko OH, Tsymbal DO, Minchenko DO, Moenner M, Kovalevska OV, Lypova NM. Inhibition of kinase and endoribonuclease activity of ERN1/IRE1 affects expression of proliferation-related genes in U87 glioma cells. Endoplasm Reticul Stress Dis 2, 18-29, 2015c.

Minchenko OH, Kryvdiuk IV, Minchenko DO, Riabovol OO, Halkin OV. Inhibition of IRE1 signaling affects expression of a subset genes encoding for TNF-related factors and receptors and modifies their hypoxic regulation in U87 glioma cells. Endoplasm Reticul Stress Dis 3, 1-15, 2016.

Minchenko OH, Luzina OY, Hnatiuk OS, Minchenko DO, Garmash YA, Ratushna OO. Expression of tumor growth related genes in IRE1 knockdown U87 glioma cells: effect of hypoxia. Ukr Biochem J 89, 40-51, 2017.

Minchenko DO, Tsymbal DO, Davydov VV, Minchenko OH. Expression of genes encoding IGF1, IGF2, and IGFBPs in blood of obese adolescents with insulin resistance. Endocr Reg 53, 34-45, 2019.

Miners JS, Love S. Endothelin-converting enzymes degrade alpha-synuclein and are reduced in dementia with Lewy bodies. J Neurochem 141, 275-286, 2017.

Nayak L, Reardon DA. High-grade gliomas. Continuum (Minneap Minn) 23, 1548-1563, 2017.

Niechi I, Silva E, Cabello P, Huerta H, Carrasco V, Villar P, Cataldo LR, Marcelain K, Armisen R, Varas-Godoy M, Fernandez C, Tapia JC. Colon cancer cell invasion is promoted by protein kinase CK2 through increase of endothelin-converting enzyme-1c protein stability. Oncotarget 6, 42749-42760, 2015.

Palmer JC, Barker R, Kehoe PG, Love S. Endothelin-1 is elevated in Alzheimer's disease and upregulated by amyloid- $\beta$. J Alzheimers Dis 29, 853-861, 2012.

Papaioannou A, Chevet E. Driving cancer tumorigenesis and metastasis through UPR signaling. Curr Top Microbiol Immunol 414, 159-192, 2018.

Russignan A, Spina C, Tamassia N, Cassaro A, Rigo A, Bagnato A, Rosano L, Bonalumi A, Gottardi M, Zanatta L, Giacomazzi A, Scupoli MT, Tinelli M, Salvadori U, Mosna F, Zamo A, Cassatella MA, Vinante F, Tecchio C. Endothelin-1 receptor blockade as new possible therapeutic approach in multiple myeloma. Br J Haematol 178, 781-793, 2017.

Semenza GL. A compendium of proteins that interact with HIF-1a. Exp Cell Res 356, 128-135, 2017.

Singh A, Happel C, Manna SK, Acquaah-Mensah G, Carrerero J, Kumar S, Nasipuri P, Krausz KW, Wakabayashi N, Dewi R, Boros LG, Gonzalez FJ, Gabrielson E, Wong KK, Girnun G, Biswal S. Transcription factor NRF2 regulates miR-1 and miR-206 to drive tumorigenesis. J Clin Invest 123, 2921-2934, 2013.

Stobdan T, Zhou D, Williams AT, Cabrales P, Haddad GG. Cardiac-specific knockout and pharmacological inhibition of Endothelin receptor type B lead to cardiac resistance to extreme hypoxia. J Mol Med (Berl) 96, 975-982, 2018.

Stow LR, Jacobs ME, Wingo CS, Cain BD. Endothelin-1 gene regulation. FASEB J 25, 16-28, 2011.

Sun RC, Denko NC. Hypoxic regulation of glutamine metabolism through HIF1 and SIAH2 supports lipid synthesis that is necessary for tumor growth. Cell Metab 19, 285-292, 2014.

Tapia JC, Niechi I. Endothelin-converting enzyme-1 in cancer aggressiveness. Cancer Lett 452, 152-157, 2019.

Teramoto K, Katoh H. The cystine/glutamate antiporter $\mathrm{xCT}$ is a key regulator of EphA2 S897 phosphorylation under glucose-limited conditions. Cell Signal 62, 109329, 2019. 
Teoh JP, Park KM, Wang Y, Hu Q, Kim S, Wu G, Huang S, Maihle N, Kim IM. Endothelin-1/endothelin A receptormediated biased signaling is a new player in modulating human ovarian cancer cell tumorigenesis. Cell Signal 26, 2885-2895, 2014.

Tsymbal DO, Minchenko DO, Hnatiuk OS, Luzina OY, Minchenko OH. Effect of hypoxia on the expression of a subset of proliferation related genes in IRE1 knockdown U87 glioma cells. Adv Biol Chem 7, 195-210, 2017.

Womeldorff M, Gillespie D, Jensen RL. Hypoxia-inducible factor-1 and associated upstream and downstream proteins in the pathophysiology and management of glioblastoma. Neurosurg Focus 37, E8, 2014.

Yalcin A, Clem BF, Imbert-Fernandez Y, Ozcan SC, Peker S, O’Neal J, Klarer AC, Clem AL, Telang S, Chesney J. 6-Phosphofructo-2-kinase (PFKFB3) promotes cell cycle progression and suppresses apoptosis via Cdk1mediated phosphorylation of p27. Cell Death Dis 5, e1337, 2014.

Yang L, Lin C, Wang L, Guo H, Wang X. Hypoxia and hypoxia-inducible factors in glioblastoma multiforme progression and therapeutic implications. Exp Cell Res 318, 2417-2426, 2012.

Zhang J, Zhao WS, Wang X, Xu L, Yang XC. Palmitic acid increases endothelin-1 expression in vascular endothelial cells through the induction of endoplasmic reticulum stress and protein kinase C signaling. Cardiology 140, 133-140, 2018.

Zhang L, Luo B, Dang YW, He RQ, Chen G, Peng ZG, Feng ZB. The clinical significance of endothelin receptor type B in hepatocellular carcinoma and its potential molecular mechanism. Exp Mol Pathol 107, 141-157, 2019.

Zhao S, Cai J, Li J, Bao G, Li D, Li Y, Zhai X, Jiang C, Fan L. Bioinformatic profiling identifies a glucose-related risk signature for the malignancy of glioma and the survival of patients. Mol Neurobiol 54, 8203-8210, 2017. 